\title{
A qualitative study of workplace facilities and employee perceived barriers, motivators and attitudes to regular physical activity in the workplace
}

\author{
L.B. Kirwan ${ }^{1}$, C.L. Fyfe ${ }^{2}$ and A.M. Johnstone ${ }^{2}$ \\ ${ }^{1}$ University College Dublin, Belfield, Dublin 4, Ireland and ${ }^{2}$ The Rowett Institute of Nutrition and Health, University of \\ Aberdeen, AB25 2ZD, UK
}

With people now spending up to 40 years of their lives in the workplace ${ }^{1}$ incorporating regular physical activity (PA) into the working day seems an opportunistic way to increase activity levels and reduce workplace health inequalities. The aim of the present study was to evaluate employee attitudes and to assess workplace facilities and attitudes towards regular PA in the workplace. A short questionnaire was designed using Survey Monkey online software. An electronic link to the survey was e-mailed to previous study participants (NeuroFAST, www.neurofast.net) and advertised on University websites and targeted social media. This study was approved by the local Ethics Committee.

The questionnaire: Q1-3 asked respondents to identify personal and environmental workplace barriers and grade each as either no barrier, a minor, medium or major barrier; Q4 evaluated personal promoting factors as being either no influence, a minor, medium or major influence on PA levels; Q5\&6 asked respondents to rate 14 workplace changes and programmes on how effective they believed each would be on promoting regular PA in the workplace, with 1 being no effect whatsoever and 5 being a major positive effect; Q7\&8 asked respondents to rate their current workplace's activity facilities and programmes as being N/A, terrible, bad, neutral, good or excellent; Q9 assessed current PA levels (150 minutes moderate or 75minutes vigorous exercise/wk in bouts of at least 10 continuous minutes) and Q10 determined weekly activity patterns.

520 responses were analysed using Survey Monkey and Microsoft Excel. Less than $15 \%$ participated in PA midweek during working hours and $35 \%$ did not meet current PA recommendations. Lack of time was the only major personal barrier reported and work commitment was seen as the most prevalent environmental barrier. Over $50 \%$ of participants reported health programmes, classes, onsite gym and equipment in their workplace as bad, terrible or N/A. Over $91 \%$ of respondents reported weight loss/maintenance, routine, social aspect and being able to walk/cycle to work/shops as promoting influences. Attitudes towards PA in the workplace were found to be optimistic with over $60 \%$ perceiving co-workers and employer's attitudes as neutral, good or excellent. Time allocation to activity, flexible working hours, workplace incentives and onsite gym/gym equipment were deemed potentially most influential on increasing activity levels.

In summary, these data suggest that the workplaces evaluated were insufficiently adapted for activity during working hours with inadequate PA facilities and programmes to support staff. Environmental barriers outweighed personal barriers; attitudes were found to be favourable towards activity and intervention strategies were deemed potentially effective if implemented. The potential to create a working environment inclusive of regular PA was recognised as viable and in order to reduce health inequalities in the workplace evidence based intervention and robust evaluation is required.

The work is funded by the Scottish Government and European Union Seventh Framework Programme (FP7/2007-2013) under grant agreement ${ }^{\circ}$ 245009 for the 'NeuroFAST' grant.

1. BUPA (2009) Healthy Work: Challenges and Opportunities to 2030, BUPA, London. 